\title{
Almacenamiento de arroz: influencia en la inocuidad del grano
}

\author{
Rice storage: effects on grain safety
}

Armazenamento de arroz: influência na segurança dos grãos

RECIBIDO: 16/9/2019 $\rightarrow$ APROBADO: 27/11/2019 \asanchez@latitud.org.uy

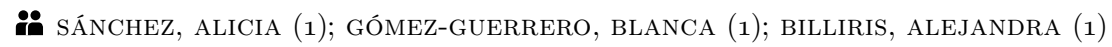

(1) LATitud - FUNDACión LATU, MONTEVIDEO, URUGUAY

\section{RESUMEN}

La producción de arroz (Oryza sativa) ocupa el quinto lugar en las exportaciones agrícolas de Uruguay. Inadecuadas condiciones de temperatura y humedad favorecen el desarrollo de hongos e insectos impactando en la inocuidad del arroz almacenado. El objetivo de este trabajo fue evaluar la sanidad del arroz cáscara en condiciones de almacenamiento en silos comerciales durante dos zafras. Se utilizó la variedad de grano largo "El Paso 144" durante 8,5 y 7 meses en los años 2017 y 2018, respectivamente. Se detectaron e identificaron agentes de biodeterioro; se determinó capacidad germinativa, recuento microbiano y contenido de toxinas en arroz extraído de las zonas superior e inferior del silo, a distintos tiempos de almacenamiento. La población microbiana disminuyó en ambos años y posiciones dentro del silo. Se identificaron principalmente dos géneros micotoxigénicos, Fusarium sp. y Aspergillus sp. En relación con micotoxinas no se detectó Zearalenona ni Ocratoxina A, y Deoxinivalenol se detectó en un máximo 120 ppb (zafra 2017). No se dectaron aflatoxinas B1, B2, G1 y G2. Los insectos identificados fueron Sitophilus sp. y Rhizopertha sp. con una incidencia máxima de 0,1\%. Estos resultados demuestran que las condiciones de almacenamiento no afectaron la seguridad del grano.

PALABRAS ClAVE: Oryza sativa, microorganismos, micotoxinas, insectos.

\section{ABSTRACT}

Rice (Oryza sativa) production ranks fifth in Uruguay's agricultural exports. Inadequate temperature and humidity conditions increase grain respiration and favor the action of insects and fungi. This process contributes to mass grain losses and stored rice biodeterioration. The objective of this work was to evaluate paddy rice sanitary conditions during storage in commercial silos through two years. The study was carried out using the "El Paso 144" long grain variety for 8,5 and 7 months in 2017 and 2018, respectively. Main biodeterioration agents were detected 
and identified. Germination capacity, microbial population and toxin content in rice extracted from the upper and lower areas of the silo were determined at different storage times. The microbial population decreased after storage time and in both silos sampling positions. Two mycotoxigenic genera, Fusarium sp. and Aspergillus sp. were detected. Mycotoxins, Zearalenone and Ocratoxin-A were not detected, while Deoxinivalenol reached a maximum level of 120 ppb (2017). Aflatoxins B1, B2, G1 and G2 were not detected. The coleopteran Sitophilus sp. and Rhizopertha sp. were identified and quantified with a maximum incidence of $0,1 \%$. These results demonstrate that storage conditions did not affect grain safety.

KEYWORDs: Oryza sativa, microorganisms, mycotoxins, insects.

\section{RESUMO}

A produção de arroz (Oryza sativa) ocupa o quinto lugar nas exportações agrícolas do Uruguai. Condições inadequadas de temperatura e umidade favorecen a ação de insetos e fungos impactando na saúde do grão. O objetivo do trabalho foi avaliar o estado sanitário de casca de arroz em condições de armazenamento em silos comerciais para duas safras. O estudo foi realizado com a variedade de grão longo "El Paso 144" por 8,5 e 7 meses em 2017 e 2018, respectivamente. Os agentes de biodeterioração foram detectados e identificados e a capacidade de germinação, a contagem microbiana e o conteúdo de toxinas no arroz extraído das áreas superior e inferior do silo foram determinados em diferentes tempos de armazenamento. A população microbiana diminuiu nos dois anos e nas posições dentro do silo. Dois gêneros micotoxigênicos foram identificados principalmente: Fusarium sp. e Aspergillus sp. Em relação às micotoxinas, Zearalenona e Ocratoxina-A não foram detectadas, enquanto o Deoxinivalenol foi detectado no máximo em 120 ppb (safra 2017). Não foram prescritas aflatoxinas B1, B2, G1 e G2. Os insetos Sitophilus sp. e Rhizopertha sp. foram detectados com uma incidência máxima de 0,1\%. Portanto, as condições de armazenamento não afetaram a segurança do grão.

PALAVRAS-CHAVE: Oryza sativa, microrganismos, micotoxinas, insetos.

\section{INTRODUCCIÓN}

En Uruguay el 95\% del volumen de grano de arroz cosechado se exporta, por lo que se ha tenido que desarrollar y mantener un sistema competitivo que satisfaga estándares altos de calidad y rendimiento. En 2019 se posicionó en el quinto lugar del ranking de productos exportados con un total de US\$ 49 millones y una participación de un $6 \%$ en el producto bruto interno del país (Salgado, 2018; Uruguay XXI, 2019).

Cuando el grano está maduro se realiza la cosecha y se conduce el grano inmediatamente a las distintas plantas de recibo para su limpieza, secado y posterior conservación. El grano ingresa a la planta industrial con una humedad de entre 24 y $16 \%$ aproximadamente, por lo que necesita, luego de una pre-limpieza, ser secado hasta alcanzar una humedad inferior a $13 \%$, de modo de asegurar un almacenamiento 
seguro. Luego se procede a una segunda etapa de limpieza del grano y se almacena en galpones, silos, silos bolsa, etcétera, para su posterior elaboración.

Cuando las condiciones en las que transcurre la fase de almacenamiento de granos son desfavorables se producen pérdidas económicas que se atribuyen a la merma de masa, calidad y valor comercial, que condicionan la disponibilidad de alimentos y sus volúmenes exportables (De Lucia y Assennato, 1993).

El establecimiento de agentes de biodeterioro en los granos almacenados depende de las condiciones ambientales (fundamentalmente humedad y temperatura), actividad de agua (aw), disponibilidad de alimento, refugio y presencia y abundancia de los competidores, depredadores y parásitos (Jian y Jayas, 2012).

De acuerdo con Abadía y Bartosik (2013), la tasa de respiración de los granos y agentes biológicos como insectos y los microorganismos presentes en los granos almacenados involucran el agua. Cuando la humedad relativa del espacio intergranario es inferior al $67 \%$ la mayoría de los hongos del almacenaje no pueden sobrevivir en la masa de granos, por lo que esta es la "humedad relativa de almacenamiento seguro". Asimismo, la variable tiempo de almacenamiento también es importante y puede definirse un "tiempo de almacenamiento seguro", entendido como el tiempo máximo que puede ser almacenado un grano a determinadas condiciones de temperatura, humedad y porcentaje de granos dañados mecánicamente, sin perder su condición de grado. Tanto la humedad relativa como el tiempo de almacenamiento seguro dependen del grano de que se trate.

La variable asociada a la humedad y que también influye en el deterioro de granos y alimentos es la actividad de agua disponible para desarrollo de microorganismos y reacciones enzimáticas. Valores cercanos a 1,0 son más propicios para el desarrollo fúngico (Cahagnier, et al., 1993).

Los hongos responsables del deterioro de los granos desde un punto de vista ecofisiológico se pueden agrupar en dos categorías: "hongos de campo", que infectan los granos antes de la cosecha, y los "hongos de almacenamiento", que generalmente son saprofitos y se desarrollan después de la cosecha (Christensen y Kaufmann, 1965). Según otros autores, podría incorporarse un tercer grupo fúngico intermedio que se compondría de hongos que continúan desarrollándose durante el almacenamiento si la aw permanece alta. Este grupo incluye especies de los géneros Cladosporium, Fusarium y Trichoderma (Mannaa y Kim, 2017). Neninger et al. (2003) reportaron más de 99 especies y 59 géneros de hongos asociados al arroz. Los géneros comúnmente detectados son: Alternaria, Epicoccum, Bipolaris, Curvularia y Cladosporium.

Los géneros que aparecen más comúnmente en almacenamiento son Fusarium, Aspergillus y en menor medida Penicillium (Richardson, 1996; Tonon, et al., 1997; Lima, et al., 2000; Gutiérrez, et al., 2002; Mew y Gonzales, 2003; Neninger et al., 2003; Karunakara y Manonmani, 2009; Pinciroli, et al., 2013; Shiratori, et al., 2017).

Otro aspecto relevante para asegurar la inocuidad del grano vinculado con el crecimiento de hongos es la presencia de micotoxinas. Se producen pérdidas económicas significativas $(25 \%)$ en los productos agrícolas mundiales debido a la contaminación por micotoxinas (Marin, et al., 2013). Estas son metabolitos secundarios producidos por estos organismos en respuesta a estrés causado por extremos 
ambientales, escasez de alimentos o competencia de otros microorganismos. Las toxinas pueden ser nefrotóxicas, inmunosupresoras, cancerígenas y teratogénicas para salud animal y humana. Los grupos más importantes de micotoxinas que se encuentran en los alimentos son: aflatoxinas, producidas por Aspergillus; ocratoxina A, producida tanto por Aspergillus como por Penicillium; tricotecenos tipo A: toxinas HT-2 y T-2 y tipo B: deoxinivalenol (DON) y nivalenol (NIV), zearalenona (ZEA), fumonisinas B1 y B2 producidas principalmente por las especies de Fusarium; alcaloides del ergot producidos por Claviceps, y altenueno, alternariol, alternariolmetil éter, altertoxina y ácido tenuazónico producidos por especies de Alternaria (Lima, et al., 2000; Marin, et al., 2013).

La proliferación de insectos en los recintos de almacenamiento es también causa de deterioro de granos y consecuentes pérdidas económicas. Respecto del riesgo de daño por insectos, las temperaturas superiores a $25{ }^{\circ} \mathrm{C}$ se consideran como de riesgo elevado para el desarrollo de insectos, entre 17 y $25^{\circ} \mathrm{C}$ de riesgo moderado, y temperaturas menores a $17^{\circ} \mathrm{C}$ corresponden a un bajo riesgo de infestación (Food and Agricultural Organization, 1994; Abadía y Bartosik, 2013). Los insectos plaga y ácaros de los granos almacenados ocasionan daños directos, por el consumo y contaminación del producto, e indirectos, como el calentamiento del grano, desarrollo de hongos y micotoxinas. Se reconocen aproximadamente 250 especies de insectos que atacan a granos y sus productos en la etapa de almacenaje, y alrededor de 20 son las especies más relevantes (Dell'Orto y Velázquez, 1985).

Los mayores perjuicios económicos son provocados por coleópteros, entre ellos Sitophilus oryzae, (Curculionidae), "gorgojo del arroz", Rhyzopertha dominica (Fabricius) (Bostrichidae) ("taladrillo de los cereales"). Se consideran plagas primarias debido a que cumplen su ciclo biológico en el interior del grano que infestan. Las que se alimentan de granos dañados, quebrados y harina se consideran plagas secundarias, como Tribolium, los lepidópteros Sitotroga cerealella (Gelechiidae), Plodia interpunctella Hübnery, Ephestia = Anagasta kuehniella, entre otros (Lorini, et al., 2009; Hagstrum, et al., 2013).

La investigación en relación con el cultivo de arroz en Uruguay se ha focalizado principalmente en el desarrollo de nuevas variedades de arroz que mejoran las características de ecofisiología, rendimiento, manejo y ciclo biológico de plagas en la etapa del cultivo de arroz (Zorrilla, et al., 2017). Sin embargo, con respecto al conocimiento del ecosistema que constituye el grano almacenado en silo no se han desarrollado estudios a nivel nacional que profundicen en el estudio de los factores bióticos y abióticos que interactúan en este ambiente. La inocuidad y calidad son aspectos decisivos para permanecer en los mercados donde se exporta este grano, por lo que estas características deben ser preservadas durante el almacenamiento (Queheille, 2018).

El objetivo del trabajo fue evaluar la sanidad del arroz cáscara en condiciones de almacenamiento en silos comerciales durante dos zafras. Como objetivos específicos se propuso determinar si existen diferencias en la inocuidad del grano en la parte superior e inferior del silo y determinar el contenido de hongos, micotoxinas e insectos. 


\section{MATERIALES Y MÉTODOS}

\section{Materiales}

Se utilizó la variedad de grano largo "El Paso 144", proveniente de un establecimiento industrial en el este de Uruguay.

\section{Muestreos en silos}

La evaluación del almacenamiento se realizó en dos silos comerciales durante 8,5 y 7 meses en las zafras 2016/2017 (mayo a enero) y 2017/2018 (junio a enero), respectivamente. El momento de los muestreos fue inicial, a los 3, 5, 7 y 8,5 meses (en la primera zafra) desde el llenado del silo. En cada fecha de muestreo se realizaron tomas embolsando $3 \mathrm{~kg}$ de arroz cáscara en nueve puntos del silo de la parte superior, seleccionados para abarcar la mayor parte de la superficie de grano. Posteriormente, de la zona de descarga media del silo se sacaron nueve bolsas de $3 \mathrm{~kg}$ de arroz en forma intermitente desde la cascada de grano. En cada fecha de muestreo se midió la temperatura de masa de grano con termocuplas.

\section{Procesamiento de muestras}

En planta se mezclaron las nueve bolsas procedentes de arriba y abajo de los silos para obtener una muestra compuesta homogénea. Se fracciona esta muestra en tres bolsas con $3 \mathrm{~kg}$ de arroz cada una de la parte inferior y superior del silo, de la que finalmente se extrae $1 \mathrm{~kg}$ y apartan $100 \mathrm{~g}$ para evaluar insectos presentes y otros $100 \mathrm{~g}$ para detectar la presencia e incidencia de hongos.

Se determinó la actividad de agua (aw) mediante equipo Aqualab y de acuerdo con el método ISO 21807 (International Organization for Standarization, 2004). La capacidad germinativa se realizó de acuerdo con el método por vitascopio 7-010 indicado por las Reglas ISTA (International Seed Testing Association, 2019). El recuento de hongos y levaduras se realizó de acuerdo al método de APHA (American Public Health Association, 2015) basado en cultivar en placas de Petri con medio de cultivo diluciones decimales sucesivas de una suspensión inicial de esporas para posteriormente realizar el conteo de unidades formadoras de colonias por gramo (UFC/g). El análisis estadístico comparativo de las muestras se realiza en función de logaritmo en base 10 del número de colonias registrado para cada muestra.

Se determinó el género de los hongos en 100 granos de arroz de la muestra compuesta de cada posición del silo. Los granos se esterilizaron superficialmente con una dilución de hipoclorito de sodio al $1 \%$ en agua destilada estéril y se ubicaron en placas de Petri (10 granos por placa) con papel filtro humedecido en el fondo de cada placa. Los granos se incubaron a $25{ }^{\circ} \mathrm{C}$ durante 5 días en alternancia de luz-oscuridad. Se aislaron los hongos emergentes a partir del grano para la determinación macro y micromorfológica de los géneros presentes (Barnett y Hunter, 1972; Domsch, et al., 1980; Mew y Gonzáes, 2002; Klich, 2002; Leslie y Summerell 
2006). En el caso de Fusarium, para obtener una aproximación de la identificación se realizó la amplificación y secuenciación de la región ITS1-5.8SrADN-ITS2K, usando los primers ITS1 e ITS4 (Nilsson, 2012).

En la zafra 2016/2017 se analizaron las siguientes micotoxinas: aflatoxinas B1, B2, G1 y G2, OTA, ZEA y DON, utilizando cromatografía líquida de alta resolución (HPLC), métodos 970.45, 995.15, 991.31, 994.08, 986.17 (AOAC International, 2016a, 2016b, 2016c, 2016d, 2016e).

En 2017 / 2018 se consideró importante conocer la existencia de micotoxinas sin dirigir el análisis a una en particular y se realizó el análisis de micotoxinas por multimétodo por LC-MSMS (cromatografía líquida masa-masa). Las micotoxinas analizadas fueron: $3+15$ acetyldeoxynivalenol, aflatoxina $\mathrm{B} 1$, aflatoxina $\mathrm{B} 1+\mathrm{B} 2+\mathrm{G} 1+\mathrm{G} 2$, aflatoxina B2, aflatoxina G1, aflatoxina G2, cytochalasina E, deoxynivalenol (DON), fumonisina B1 + B2, nivalenol, ochratoxina-A (OTA), T2 \& toxina HT2 y Zearalenona.

A partir de los $100 \mathrm{~g}$ de cada submuestra se detectaron los insectos adultos presentes (Trochez, 1987; Fourar-Belaifa, et al., 2011; Hagstrum, et al., 2013; USDA, 2015).

El análisis estadístico de los datos se realizó utilizando el software JMP@ 2012 y el efecto del almacenamiento se evaluó comparativamente entre periodos de muestreo y entre posiciones del silo (ANOVA).

\section{RESULTADOS}

La capacidad germinativa al final del período de almacenamiento presentó valores por encima del $90 \%$ en ambos años (Tabla 1). En 2017 no se registraron cambios, mientras que en 2018 esta variable no disminuyó al final del periodo en la parte inferior, pero en la parte superior la capacidad germinativa disminuyó en $3 \%$ aproximadamente.

TABLA 1. Capacidad germinativa (\%) del grano de arroz según posición en silo y zafra al inicio y fin del almacenamiento. D.E: desvío estandar. $\alpha=0,05$, valores seguidos de la misma letra no son estadísticamente diferentes.

\begin{tabular}{|cccccc|}
\hline & & Silo abajo & D.E & Silo arriba & D.E \\
\hline $2016 / 2017$ & Inicial & $97,7^{\mathrm{a}}$ & 0,3 & $98,0^{\mathrm{a}}$ & 0,0 \\
\hline & Final & $98,0^{\mathrm{a}}$ & 1,0 & $99,7^{\mathrm{b}}$ & 1,0 \\
\hline $2017 / 2018$ & Inicial & $86,3^{\mathrm{a}}$ & 5,7 & $98,0^{\mathrm{a}}$ & 1,0 \\
\hline & Final & $95,7^{\mathrm{b}}$ & 1,2 & $94.7^{\mathrm{b}}$ & 1,5 \\
\hline
\end{tabular}

La variable humedad de grano fluctuó a lo largo del periodo de almacenamiento. Se evidenció una pérdida de humedad del grano en la parte superior del silo, pero no en la zona inferior. 
TABLA 2. Valores de contenido de humedad del grano $(\mathrm{H} \%)$ promedio en los diferentes momentos de muestreo de acuerdo con la zafra 2016/2017 y $2017 / 2018$ y la posición en el silo. D.E: desvío estándar. $\alpha=0,05$, valores seguidos de la misma letra no son estadísticamente diferentes.

\begin{tabular}{|c|c|c|c|c|c|c|c|}
\hline & \multicolumn{3}{|c|}{$\begin{array}{c}\text { Humedad de grano (\%) } \\
\text { 2017/2018 }\end{array}$} & \multicolumn{4}{|c|}{$\begin{array}{l}\text { Humedad de grano }(\%) \\
\qquad 2016 / 2017\end{array}$} \\
\hline & Media & D.E & Media & D.E & Media & Media & D.E \\
\hline Mayo & $13,4^{\mathrm{b}}$ & 0,1 & & & $12,9^{\mathrm{a}}$ & 0,2 & \\
\hline Junio & & & $12,8^{\mathrm{b}}$ & 0,2 & & & $13,2^{\mathrm{b}}$ \\
\hline Julio & $13,2^{\mathrm{bc}}$ & 0,2 & & & $12,9^{\mathrm{a}}$ & 0,3 & \\
\hline Agosto & $13,0^{\mathrm{c}}$ & 0,1 & & & $12,6^{\mathrm{ab}}$ & 0,1 & \\
\hline Setiembre & & & $12,9^{\mathrm{b}}$ & 0,0 & & & $13,5^{\mathrm{a}}$ \\
\hline Octubre & $14,0^{\mathrm{a}}$ & 0,1 & & & $12,3^{\mathrm{bc}}$ & 0,1 & \\
\hline Diciembre & $13,1^{\mathrm{c}}$ & 0,1 & $13,1^{\mathrm{a}}$ & 0,1 & $12,0^{\mathrm{c}}$ & 0,1 & $12,9^{\mathrm{c}}$ \\
\hline Enero & $13,3^{\text {bc }}$ & 0,2 & $13,0^{\mathrm{ab}}$ & 0,0 & $12,1^{\mathrm{c}}$ & 0,3 & $12,8^{\mathrm{d}}$ \\
\hline
\end{tabular}

La actividad de agua y humedad solo aumentó significativamente en la parte inferior del silo en el primer mes respecto al final (desde 0,70 a 0,72 ) y en el $5^{\circ}$ mes de 13,4 a 14\%, respectivamente (Tablas 2 y 3), lo que no se reflejó en un incremento de la población microbiana al final del periodo en 2017 , luego de 8,5 meses de almacenamiento. Un comportamiento similar ocurrió en 2018.

TABLA 3. Valores de actividad de agua del grano promedio en los diferentes momentos de muestreo de acuerdo con la zafra 2016 / 2017 y 2017 / 2018 y la posición en el silo. D.E: desvío estándar. $\alpha=0,05$, valores seguidos de la misma letra no son estadísticamente diferentes.

\begin{tabular}{|c|c|c|c|c|c|c|c|c|}
\hline \multirow[b]{3}{*}{ Mes } & \multicolumn{4}{|c|}{ Abajo } & \multicolumn{4}{|c|}{ Arriba } \\
\hline & \multicolumn{2}{|c|}{$\begin{array}{c}\text { Actividad de } \\
\text { agua del grano } \\
2016 / 2017\end{array}$} & \multicolumn{2}{|c|}{$\begin{array}{c}\text { Actividad de } \\
\text { agua del grano } \\
2017 / 2018\end{array}$} & \multicolumn{2}{|c|}{$\begin{array}{c}\text { Actividad de } \\
\text { agua del grano } \\
2016 / 2017\end{array}$} & \multicolumn{2}{|c|}{$\begin{array}{c}\text { Actividad de } \\
\text { agua del grano } \\
2017 / 2018\end{array}$} \\
\hline & Media & D.E & Media & D.E & Media & D.E & Media & D.E \\
\hline Mayo & $0,70^{\mathrm{b}}$ & 0,00 & & & $0,64^{\mathrm{b}}$ & 0,00 & $0,70^{\mathrm{b}}$ & 0,01 \\
\hline Junio & & & $0,67^{\mathrm{c}}$ & 0,00 & & & & \\
\hline Julio & $0,62^{\mathrm{d}}$ & 0,00 & & & $0,66^{\mathrm{a}}$ & 0,01 & & \\
\hline Agosto & $0,62^{\mathrm{d}}$ & 0,01 & & & $0,62^{\mathrm{bc}}$ & 0,02 & & \\
\hline Setiembre & & & $0,73^{\mathrm{a}}$ & 0,06 & & & $0,75^{\mathrm{a}}$ & 0,00 \\
\hline Octubre & $0,68^{\mathrm{bc}}$ & 0,01 & & & $0,60^{c}$ & 0,01 & & \\
\hline Diciembre & $0,67^{\mathrm{c}}$ & 0,01 & $0,71^{\mathrm{b}}$ & 0,01 & $0,61^{\mathrm{bc}}$ & 0,00 & $0,66^{c}$ & 0,01 \\
\hline Enero & $0,72^{\mathrm{a}}$ & 0,01 & $0,70^{\mathrm{b}}$ & & $0,64^{\mathrm{bc}}$ & 0,00 & $0,68^{c}$ & 0,00 \\
\hline
\end{tabular}


El recuento microbiano en arroz cáscara disminuyó arriba y abajo al final del almacenamiento. En ambos años se observó la misma tendencia (Figura 1).

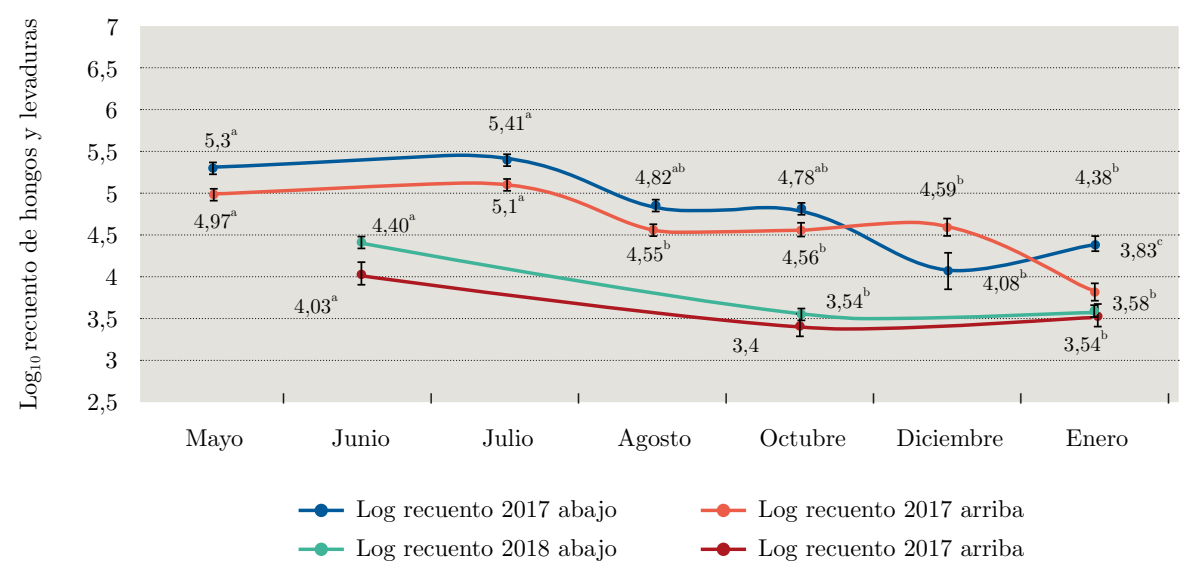

Figura 1. Valores de Logaritmo del recuento de hongos y levaduras del grano en las muestras de la parte superior e inferior del silo durante la zafra 2016 / 2017. Valores seguidos de la misma letra no son estadísticamente diferentes (test de Tukey, $\alpha=0,05$ ).

En ambos años se detectaron hongos asociados a la etapa de cultivo, como Fusarium (9\%) (Figura 2). A este aislamiento se le realizó la comparación de la secuencia obtenida región ITS1-5.8SrADN-ITS2K con la base de datos del NCBI (National Center for Biotechnology Information). Dentro de los resultados obtenidos con mayor homología (97\%, cobertura 75\%), se encontraron las especies Fusarium culmorum, Fusarium graminearum y Gibberella zeae (teleomorfo de Fusarium graminearum), las cuales son frecuentemente encontradas contaminando granos de cereales, si bien no es posible asignar con exactitud la identificación a nivel de especie de Fusarium. También se detectaron otros hongos patógenos de semillas y granos de cereales como Alternariasp. (10\%), Bipolarissp. (3\%), Curvulariasp. (13\%), Macrodochium sp. (5\%), Phoma sp. (2\%), Rhyzopus sp. (20\%), y Tilletia sp. (2\%), y hongos asociados a la etapa de almacenamiento de granos, como Aspergillus sp. (24\%) y Penicillium sp. (12\%).

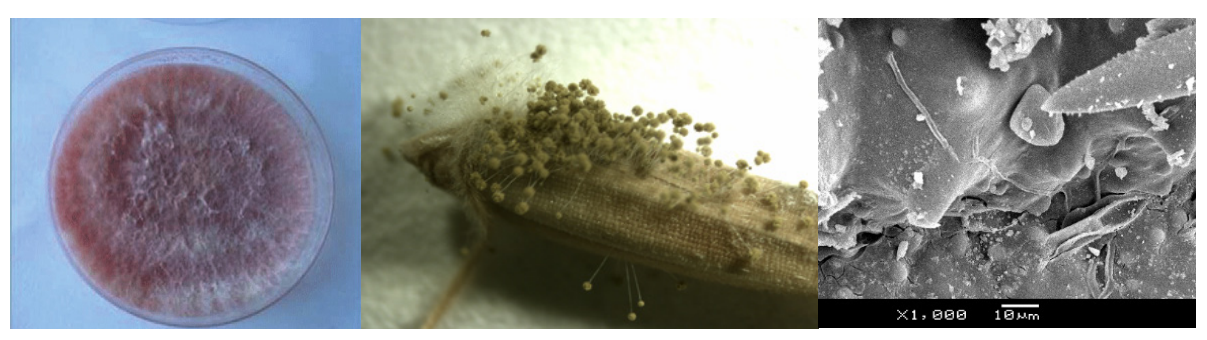

Figura 2. A) Fusarium sp. B) Aspergillus sp. (fotos Sánchez, A.).

C) Detalle de hifas sobre grano, micrografía tomada con microscopio de barrido SEM (UdelaR, Facultad de Ciencias). 
En cuanto a la detección de micotoxinas solo se detectó deoxinivalenol en ambas zafras y zeralonona en la zafra 2017/2018 (Tabla 4).

TABLA 4. Concentración (ppb) promedio de toxinas detectadas de acuerdo con la zona del silo y períodos de almacenamiento. ${ }^{1}$ valores $<$ representan los límites de detección y cuantificación.

\begin{tabular}{|c|c|c|c|c|}
\hline & \multicolumn{2}{|c|}{ Zafra $2016 / 2017^{1}$} & \multicolumn{2}{|c|}{ Zafra $2017 / 2018^{1}$} \\
\hline & Silo arriba & Silo abajo & Silo arriba & Silo abajo \\
\hline DON (Deoxinivalenol) (ppb) & 122 & 113 & 29,3 & $<20$ \\
\hline ZEA (Zearalenona) (ppb) & $<30$ & $<30$ & 12,9 & $<15$ \\
\hline OTA (Ocratoxina A) ppb & $<2,2$ & $<2,2$ & $<1,0$ & $<1,0$ \\
\hline $\begin{array}{c}\text { AFLA (Aflatoxinas:B1-B2; } \\
\text { G1- G2) ppb }\end{array}$ & $<2,6$ & $<2,6$ & $<1,0$ & $<1,0$ \\
\hline $\begin{array}{c}3+15 \text { acetildeoxynivalenol } \\
\text { ppb }\end{array}$ & - & - & $<20,0$ & $<20,0$ \\
\hline nivalenol ppb & - & - & $<50,0$ & $<50,0$ \\
\hline fumonisina $\mathrm{B} 1+\mathrm{B} 2 \mathrm{ppb}$ & - & - & $<20,0$ & $<20,0$ \\
\hline citochalasina E ppb & - & - & $<2,0$ & $<2,0$ \\
\hline T2 y HT2 -toxina ppb & - & - & $<10,0$ & $<10,0$ \\
\hline
\end{tabular}

Los insectos se identificaron mediante claves y presuntivamente pudieron asignarse a nivel de especie a Sitophylus oryzae (Curculionidae) ("gorgojo del arroz") y Rhyzopertha dominica ("barrenador o taladrillo de los granos") (Bostrichidae), los cuales se encontraron en ambas posiciones del silo (Figuras 3 y 4) y su emergencia se registró en diciembre y enero (Figura 5).

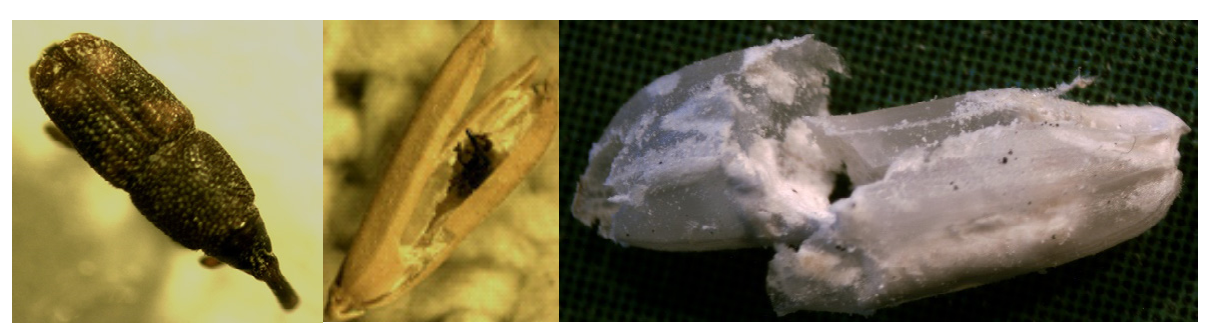

Figura 3. A) Adulto de Sitophilus oryzae. B) y C) daño en grano ocasionado por S. oryzae (fotos Sánchez, A.). 


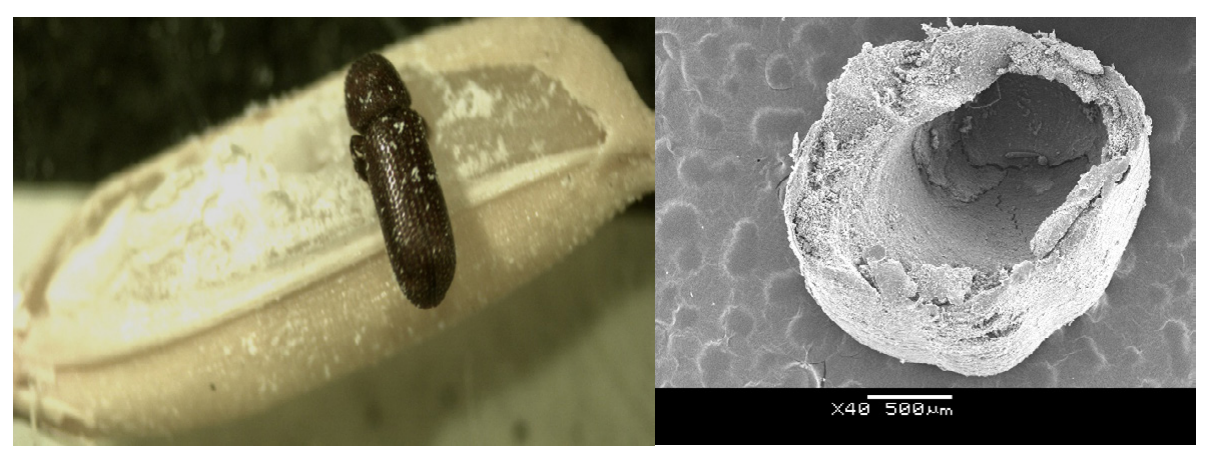

Figura 4. A) Adulto de Rhyzopertha dominica (foto Sánchez, A.).

B) Galería de alimentación de $R$. dominica, micrografía tomada con microscopio de barrido SEM (UdelaR, Facultad de Ciencias).

El recuento de adultos fue en promedio de 1 arriba y 11 abajo cada $100 \mathrm{~g}$ en 2017. En 2018, los respectivos valores, arriba y abajo, en diciembre, fueron de 4 y 9 adultos, mientras que en enero de 2019 correspondieron a 3 y 2 adultos cada $100 \mathrm{~g}$, respectivamente. El gorgojo del arroz es el que se presentó entre un 70-90\% respecto al resto de los otros insectos. El taladrillo Rhizopertha dominica se presentó en un porcentaje menor, $29 \%$, y no se detectó en 2017. Se detectaron otros insectos denominados plagas secundarias (se alimentan de grano previamente deteriorado por plagas primarias como Sitophilus y Rhyzopertha), como Plodia sp. y Tribolium sp. Estos últimos aparecieron en la zona inferior del silo y en el último muestreo de enero de 2019.

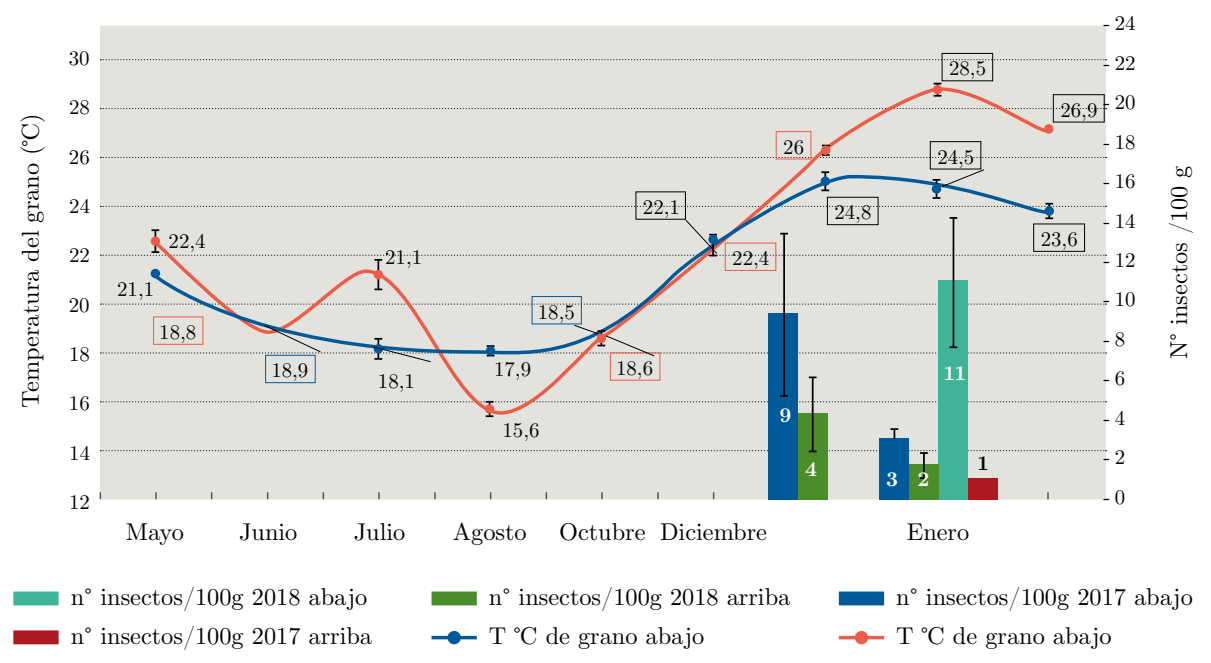

Figura 5. Valores de temperatura de las muestras de la parte superior del silo y de abajo durante los meses de almacenamiento y número de insectos emergidos. Los valores de temperatura recuadrados pertenecen a la zafra $2017 / 2018$. 
El comportamiento de la temperatura ocurrió a la inversa de lo sucedido con la humedad del grano, aumentando hacia el final del almacenamiento en ambas posiciones y zafras (Figura 5).

\section{DISCUSIÓN}

Las condiciones de manejo del ambiente silo permitieron a través de las variables estudiadas mantener la vitalidad del grano, ya que no se perdió capacidad germinativa, que superó el $95 \%$ al final del periodo de almacenamiento.

En las dos zafras se mantuvo la humedad indicada para el arroz paddy, de $13 \%$. Aun en un breve lapso, en 2017, cuando subió a 14\% y aumentó la actividad de agua, no se incrementó la población microbiana. Abadía y Bartosik (2013) reafirman la importancia de este parámetro y sugieren que si la temperatura del grano de arroz se mantiene a $25{ }^{\circ} \mathrm{C}$ y a $13 \%$ de humedad, podría almacenarse durante aproximadamente 17 meses, mientras que si la humedad aumenta a 14\%, el periodo se acortaría a 7 meses.

La humedad tampoco influyó en la actividad de agua del grano. El primer indicador potencial de deterioro de un alimento es la actividad de agua, es decir, el agua de constitución disponible para reacciones enzimáticas y crecimiento microbiano. Si bien los valores variaron al comienzo y fin del período de almacenamiento para las dos zafras estudiadas (Tabla 2), se encontraron en un rango promedio de aw 0,6 a 0,7 , en el cual el crecimiento de hongos es mínimo. Estos valores no promueven el crecimiento de Aspergillus sp. ni de Penicillium sp., cuyas actividades acuosas mínimas a las que pueden desarrollarse se sitúan en valores de aw por encima de 0,70 (Mannaa y Kim, 2017).

El valor de recuento de microorganismos se encuentra en valores normales (varió entre $10^{3}$ y $10^{5} \mathrm{UFC} / \mathrm{g}$ ), ya que los niveles de propágulos de hongos encontrados en granos y harinas de calidad alimentaria pueden variar entre aproximadamente $10^{2}$ y $10^{5} \mathrm{UFC} / \mathrm{g}$, dependiendo del tipo de grano y las condiciones de cultivo, cosecha, secado y almacenamiento (incluido el tiempo de almacenamiento) (International Commission on Microbiological Specifications for Foods, 1986).

Los hongos detectados fueron los que habitualmente se reportan en granos y en el de arroz específicamente. Los tres géneros que se detectaron en este trabajo fueron de naturaleza micotoxigénica, como Fusarium, Aspergillus y Penicillium. Asimismo, en relación con los géneros de hongos asociados al cultivo, también se detectaron generos reportados en bibliografía como Alternaria sp., Curvularia sp., Tilletia sp. y Michrodoquium sp. (Martínez, et al., 2018).

Generalmente, la infección por Fusarium sp. aparece en el campo, como la de Aspergillus sp., aunque este género puede crecer a más bajas actividades de agua, por lo que es común que prevalezca en condiciones de almacenamiento. El género Penicillium aparece con menor frecuencia infectando arroz paddy y se relaciona más estrechamente con la fase de almacenamiento (Majeed, et al., 2018; Bertuzzi, et al., 2019).

En relación con las micotoxinas, su presencia puede estar influenciada por múltiples factores como tipo de grano, variedades, manejo de cultivo y postcosecha. 
En este trabajo, en las dos zafras solo se detectó DON en concentraciones cuantificables (29 a $122 \mathrm{ppb}$ ). Los valores no revisten significancia de acuerdo con el límite admitido por Codex Alimentarius (2018) para cereales en grano trigo, maíz y cebada, que corresponde a 2.000 ppb., y de la "Regulación Europea para cereales sin procesamiento", que acepta un límite mínimo de 1250 ppb (Cheli, et al., 2014). La presencia de DON puede atribuirse a la infección a campo, donde Fusarium se desarrolla con aw cercanas a 1. En el grano permanecen las toxinas, aunque el hongo no se detecte, y también puede suceder a la inversa. De los hongos de almacenamiento se aisló Aspergillus sp. y Penicillium sp., aunque no se detectaron aflatoxinas B1, B2, G1 y G2, ni OTA.

Bertuzzi et al. (2019) obtuvieron un patrón similar al obtenido en este trabajo en el sentido de que las aflatoxinas, el deoxinivalenol y ocratoxina A no revistieron importancia. También se registraron en niveles por debajo de los de reporte o detección. Estos autores tomaron como referencia los valores mínimos admitidos por la Unión Económica Europea (Cheli, et al., 2014).

El aumento de la temperatura hacia el final del periodo de almacenamiento promovió el desarrollo de insectos. Sin embargo, la densidad de insectos no excede valores alcanzados en estudios de almacenamiento de otros cereales como trigo (Toews, et al., 2005; Flinn, et al., 2010). Se ha reportado que la morfología y composición de la cáscara de arroz le confiere una mayor protección frente a microorganismos e insectos en comparación con otros cereales como trigo, maíz o cebada (Ashamo, 2006).

La temperatura a la que la masa de granos llegó en los dos silos y en ambos años está dentro del rango óptimo de desarrollo de estos insectos, que va de $25^{\circ} \mathrm{C}$ a $35^{\circ} \mathrm{C}$.

Las especies que aparecieron, Sitophilus oryzae y Rhyzopertha dominica, son cosmopolitas y aparecen durante el estacionamiento de granos como maíz, trigo y cebada, etcétera. Trematerra et al. (2004) realizaron prospecciones de silos y plantas de procesamiento de arroz con trampas y reportaron una prevalencia de Sitophilus oryzae L. (72,3\%) sobre Rhyzopertha dominica (Fabricius) (8,6\%). Aunque la metodología de captura fue diferente, en este trabajo también se constató una predominancia de $S$. oryzae (70-90\%) sobre $R$. dominica (29\%).

Cabe señalar que el resultado presentado en cuanto a número de insectos da un valor aproximado de la densidad de población. Para determinar la proporción de grano dañado sin signos de ataque ("infestación no visible o escondida"), se deberían disectar los granos, utilizando técnicas de tinción, gravimetría y rayos X, etcétera (Xmgwei, 1998).

\section{CONCLUSIONES}

Las condiciones de almacenamiento no afectaron la capacidad germinativa del grano de arroz cáscara, que se mantuvo viable a lo largo del período.

Las condiciones de humedad del grano y temperatura no promovieron el desarrollo fúngico ni la presencia de micotoxinas. 
Los insectos se detectaron en los últimos meses de almacenamiento, lo que se asocia a aumento de la temperatura de grano.

Por esto se concluye que las condiciones de almacenamiento no afectaron la sanidad del grano de arroz cáscara desde el punto de vista fúngico, contenido de micotoxinas e insectos.

\section{RECONOCIMIENTOS}

Se agradece al LATU (Laboratorio Tecnológico del Uruguay) por la financiación del Proyecto Almacenamiento, componente del Programa de Industrialización de Arroz de Latitud.

\section{REFERENCIAS}

Abadía, B. y Bartosik, R. 2013. Manual de buenas prácticas en postcosecha de granos: hacia el agregado de valor en origen de la producción primaria. Buenos Aires: INTA. AOAC International, 2016a. Official Methods of Analysis of AOAC International. 20a ed. Gaithersburg: AOAC. Official Method 970.45, first action 1970-final action 1988. AOAC International, 2016b. Official Methods of Analysis of AOAC International. 20a ed. Gaithersburg: AOAC. Official Method 995.15, first action 1995-final action 1999. AOAC International, 2016c. Official Methods of Analysis of AOAC International. 20a ed. Gaithersburg: AOAC. Official Method 991.31, first action 1991-final action 1994. AOAC International, 2016d. Official Methods of Analysis of AOAC International. 20a ed. Gaithersburg: AOAC. Official Method 994.08, first action 1994-final action 1997. AOAC International, 2016e. Official Methods of Analysis of AOAC International. 20a ed. Gaithersburg: AOAC. Official Method 986.17, first action 1986-final action 1990. American Public Health Association, 2015. Yeast and moulds. En: American Public Health Association. Compendium of methods for the microbiological examination of foods. Cap. 21. Washington: APHA.

Ashamo, M.O., 2006. Relative susceptibility of some local and elite rice varieties to the rice weevil, Sitophilus oryzae L. (Coleoptera: Curculionidae) En: Journal of Food, Agriculture \& Environment, 4(1), pp.249-252.

Barnett, H.L. y Hunter, B., 1972. Illustrated genera of imperfect fungi. Minneapolis: Burguess Bublishing Company.

Bertuzzi, T., Romani, M., Rastelli, S. y Giorni, P., 2019. Mycotoxins and related fungi in italian paddy rice during the growing season and storage. En: Toxins, 11(3), pp.151.

Cahagnier, B., Lesage, L. y Richard-Molard, D., 1993. Mould growth and conidiation in cereal grains as affected by water activity and temperature. En: Letters in Applied Microbiology, 17(1) pp. 7-13.

Cheli, F., Battaglia, D., Gallo, R., y Dell'Orto, V., 2014. EU legislation on cereal safety: An update with a focus on mycotoxins. En: Food Control, 37, pp.315-325.

Codex Alimentarius, 2018. General standard for contaminants and toxins in food and feed CXS 193-1995. Adopted in 1995, revised in 1997, 2006, 2008, 
2009, amended in 2010, 2012, 2013, 2014, 2015, 2016, 2017, 2018, 2019

[En línea]. Roma: Codex Alimentarius. [Consulta: 3 de setiembre 2019].

Disponible en: http://www.fao.org/fao-who-codexalimentarius/sh-proxy/ es $/ ? \operatorname{lnk}=1 \&$ url $=$ https $\% 253 \mathrm{~A} \% 252 \mathrm{~F} \% 252 \mathrm{Fworkspace}$.fao.org $\% 252 \mathrm{Fsites} \% 252 \mathrm{Fcod}$ ex\%252FStandards\%252FCXS\%2B193-1995\%252FCXS_193e.pdf

International Commission on Microbiological Specifications for Foods, 1986.

Microorganisms in Foods, 2. Sampling for microbiological analysis. Principles and specific applications [En línea]. 2a ed. [s.l.]: Blackwell Scientific Publications. [Consulta: 2 de setiembre de 2019]. Disponible en: https://seafood.oregonstate. edu/sites/agscid7/files/snic/sampling-for-microbiological-analysis-principles-andspecific-applications-icmsf.pdf

Christensen, C.M. y Kaufmann, H., 1965. Deterioration of stored grains by fungi. En: Annual Review of Phytopathology, 3, pp.69-84.

De Lucia, M. y Assennato, D., 1993. La ingeniería en el desarrollo. Manejo y tratamiento de granos poscosecha [En línea]. Roma: FAO. [Consulta: 20 de Agosto de 2019]. Disponible en: http://www.fao.org/3/x5041s/x5041s00.htm

Dell'Orto, T.H. y Arias Velázquez, C.J., 1985. Insectos que dañan granos productos almacenados. Santiago: FAO. (Tecnología Poscosecha, 4). [Consulta: 2 de setiembre de 2019]. Disponible en: http://www.fao.org/3/x5053S/x5053s00.htm

Domsch, K.H., Gams W. y Anderson, T.H., 1980. Compendium of soil fungi. Vol. 2. Londres: Academic Press.

Flinn, P. W., Hagstrum, D. W., Reed, C. y Phillips, T.W., 2010. Insect population dynamics in commercial grain elevators. En: Journal of Stored Products Research, 46(1), pp.43-47.

Food and Agricultural Organization, 1994. Grain storage techniques. Evolution and trends in developing countries [En línea]. Roma: FAO. (FAO Agricultural Services Bulletin, $\left.N^{\circ} 109\right)$. [Consulta: 2 de setiembre de 2019]. Disponible en: http://www. fao.org/3/T1838E/T1838E1f.htm

Fourar-Belaifa, R., Fleurat-Lessard, F. y Bouznad, Z., 2011. A systemic approach to qualitative changes in the stored-wheat ecosystem: prediction of deterioration risks in unsafe storage conditions in relation to relative humidity level, infestation by Sitophilus oryzae (L.), and wheat variety. En: Journal of Stored Products Research, 47, 1, pp.48-61.

Gutiérrez, S.A., Mazzanti De Castañón, M.A. y Cúndom, M.A., 2002. Hongos presentes en semillas de arroz del noreste de Argentina. En: Fitopatología, 37, pp.156-163

Hagstrum, D., Klejdysz, T., Subramanyam y Bhadriraju Nawrot, J., 2013. Atlas of stored-product insects and mites. Washington: AACC, INC. 589 p.

International Seed Testing Association, 2019. International rules for seed testing. Validated seed health testing methods [En línea]. Bassersdorf: ISTA. Method 7-010, 2019. [Consulta: 3 de setiembre]. Disponible en: https://www.seedtest.org/upload/ cms/user/ISTARules2019SHmethods7-010.pdf

International Organization for Standarization, 2004. ISO 21807: Microbiology of food and animal feeding stuffs -- Determination of water activity. Ginebra: ISO. 
Jian, F. y Jayas, S., 2012. Ecosystem approach to grain storage. En: Agriculture Research, 1(2), pp.148-156

KarunakaraK., E.R., Murthy, R., Manonmani, H.K., 2009. Incidence of fusarium toxins in rice from Karnataka, India. En: Research Journal of Toxins, 1, pp.1-7.

Klich, M.A., 2002. Identifcation of common Aspergillus species. Utrecht:

Centraalbureau voor Schimmelcultures.

Leslie, J. y Summerell, B., 2006. The Fusarium laboratory manual. Iowa: Blackwell publishing.

Lima, C.L., Orsi, R.B., Dilkin, P. y Correa, B., 2000. Mycoflora and aflatoxigenic species in derivatives of milled rice. En: Ciência e Tecnologia de Alimentos, 20, pp.37-39.

Lorini, I., Krzyzanowski, F., de Barros França-Neto, J. y Henning, A., 2009. Principais pragas e métodos de controle em sementes durante o armazenamento. Brasilia: Abrasem.

Majeed, S., De Boevre, M., De Saeger, S., Rauf, W., Tawab, A., Fazal-e-Habib e Iqbal, M., 2018. Multiple mycotoxins in rice: occurrence and health risk assessment in children and adults of Punjab, Pakistan. En: Toxins, 10(2), p. 77.

Mannaa, M. y Kim, K.D., 2017. Influence of temperature and water activity on deleterious fungi and mycotoxin production during grain storage.

En: Mycobiology, 45(4), pp.240-254.

Marin, S., Ramos, A.J., Cano-Sancho G. y Sanchis, V., 2013. Mycotoxins: occurrence, toxicology, and exposure assessment. En: Food and Chemical Toxicology, 60, pp.218-237.

Martínez, S., Bao, L. y Escalante, F., 2018. Manual de identificación de enfermedades y plagas en el cultivo de arroz. Montevideo: INIA. (Boletín de Divulgación, $\mathrm{N}^{\circ} 116$ ).

Mew,T.W. y Gonzales, P., 2002. A handbook of rice seedborne fungi. Los Baños: International Rice Research Institute. 83p.

Neninger, L.H., Hidalgo, E.I., Barrios, L.M. y Pueyo, M., 2003. Hongos presentes en semillas de arroz (Oryza sativaL.) en Cuba. En: Fitosanidad, 7, pp.7-11.

Nilsson, R., Tedersoo, L., Abarenkov, K., Ryberg, M., Kristiansson, E., Schoch, C., Nylander, J., Bergsten, J., Porter, T., Jumpponen, A., Vaishampayan, P., Ovaskainen, O. Hallenberg, N., Bengtsson-Palme, J., Eriksson, K., Larsson, K-H., Larsson, E. y Koeljalg, U., 2012. Five simple guidelines for establishing basic authenticity and reliability of newly generated fungal ITS sequences. En: MycoKeys, 4, pp.37-63.

Pinciroli, M., Gribaldo, A., Vidal, A., Bezus, R. y Sisterna, M., 2013. Mycobiota evolution during storage of paddy, brown and milled rice in different genotypes. En: Summa Phytopathologica, 39(3), pp.157-16.

Queheille, N., 2018. Inocuidad: un atributo decisivo para el arroz [En línea]. En: Arroz, setiembre 2018. pp.54-56. [Consulta: 3 de setiembre de 2019]. Disponible en: http://www.aca.com.uy/wp-content/uploads/2017/08/INOCUIDAD.pdf

Richardson, J.M., 1996. Seed mycology. En: Mycological Research, 100(4), pp.385-392.

Salgado, L., 2018. Arroz: situación y perspectivas. En: OPYPA. Anuario OPYPA. Montevideo: MGAP. pp. 215-227. [Consulta: 29 agosto 2019]. Disponible en: http://www.mgap.gub.uy/unidad-organizativa/oficina-de-programacion-y- 
politicas-agropecuarias/publicaciones/anuarios-opypa/2018

Shiratori, N., Kobayashi, N., Tulayakul, P., Sugiura, Y., Takino, M., Endo, O. y Sugita-Konishi, Y., 2017. Occurrence of Penicillium brocae and Penicillium citreonigrum, which produce a mutagenic metabolite and a mycotoxin citreoviridin, respectively, in selected commercially available rice grains in Thailand. En: Toxins, 9(6), E194.

Toews M., Thomas, P. y Payton, M., 2005. Estimating populations of grain beetles using probe traps in wheat-filled concrete silos. En: Environmental Entomology, 34(3), pp.712-718.

Tonon, S.A., Marucci, R.S., Jerke, G. y García, A., 1997. Mycoflora of paddy and milled rice produced in the region Northeastern Argentina and Southern Paraguay. En: International Journal of Food Microbiology, (37), pp.231-235.

Trematerra, P.P., Sciarretta, M.C.Z. y Lazzari, A.S.M.N., 2004. Spatio-temporal analysis of insect pests infesting a paddy rice storage facility. En: Neotropical Entomology, 33(4), pp.469-479.

Trochez, A., 1987. Manual de reconocimiento de insectos asociados con productos almacenados. Bogotá: IICA. (Serie Miscelánea, 31).

Uruguay XXI, 2019. Informe mensual de comercio exterior, Agosto de 2019 [En línea]. Montevideo: Uruguay XXI. [Consulta: 13 de Setiembre, 2019]. Disponible en: https:// www.uruguayxxi.gub.uy/es/centro-informacion/informes-de-comercio-exterior/

USDA, 2015. Stored grain reference. Washington: USDA. 74p.

Xmgwei, H., Xianchang, T. y Jianchun, W., 1998. The comparison of five determination methods for hidden insect infestation. En: Proceedinqs of the 7th International World Conference on Stored-product Protection. Vol. 2. Beijing: Sichuan Publishing House of Science and Technology. pp.14-19.

Zorrilla, G., Martinez, S. y Saravia, H., eds, 2017. Arroz 2017. Montevideo: INIA. (INIA Serie Técnica, 233). 132 p. ISBN 978-9974-38-381-4. 